$\mathrm{J}$ o u r n a l of

Mathematics

and Applications

JMA No 44, pp 93-105 (2021)

\title{
On Multiplicative (Generalized)-Derivations and Central Valued Conditions in Prime Rings
}

\author{
Gurninder S. Sandhu, Ayşe Ayran and Neşet Aydin
}

\begin{abstract}
Let $R$ be a prime ring with multiplicative (generalized)derivations $(F, f)$ and $(G, g)$ on $R$. This paper gives a number of central valued algebraic identities involving $F$ and $G$ that are equivalent to the commutativity of $R$ under some suitable assumptions. Moreover, in order to optimize our results, we show that the assumptions taken cannot be relaxed.
\end{abstract}

AMS Subject Classification: 16N60, 16W20.

Keywords and Phrases: Prime ring; Multiplicative generalized derivation; One-sided ideal; Commutativity.

\section{Introduction}

All through this paper, $R$ will always denote an associative prime ring, unless otherwise mentioned and $Z(R)$ denotes the center of $R$. For any $x, y \in R$, the commutator $x y-y x$ is denoted by the symbol $[x, y]$. It is well-known that a ring $R$ is said to be prime if for any $a, b \in R ; a R b=(0)$ implies either $a=0$ or $b=0$ and it is called semiprime if $a R a=(0)$ implies $a=0$. By a multiplicative derivation, we mean a mapping $\delta: R \rightarrow R$ (not necessarily additive) satisfying the relation $\delta(x y)=\delta(x) y+x \delta(y)$ for all $x, y \in R$. If $\delta$ is necessarily additive, then it is called a derivation. Therefore every derivation is a multiplicative derivation but the converse is not generally true; for example, let $R=C[0,1]$ be the ring of all continuous (real or complex valued) functions and define a map $\delta: R \rightarrow R$ such that

$$
\delta(f)(x)= \begin{cases}f(x) \log |f(x)|, & \text { when } f(x) \neq 0 \\ 0, & \text { otherwise. }\end{cases}
$$

COPYRIGHT (C) by Publishing House of Rzeszów University of Technology P.O. Box 85, 35-959 Rzeszów, Poland 
An additive mapping $F: R \rightarrow R$ is called a generalized derivation if there exists a derivation $\delta$ of $R$ such that $F(x y)=F(x) y+x \delta(y)$ for all $x, y \in R$. Then it is natural to think of the unified notion of generalized derivation and multiplicative derivation. Recently, Dhara and Ali [7] introduced a mapping called multiplicative (generalized)-derivation viz., a mapping $F: R \rightarrow R$ is called a multiplicative (generalized)-derivation if there exists a function $f: R \rightarrow R$ such that $F(x y)=F(x) y+x f(y)$ for all $x, y \in R$, where $F$ and $f$ are not necessarily additive. However, these mappings appeared first time in a paper by Gusić [9]. Later on it is figured out that the associated function of a multiplicative (generalized)-derivation is a multiplicative derivation (see [6]).

During the last six decades there have been many results showing that the global structure of a ring is often tightly connected with the behaviour of additive and multiplicative mappings defined on it (see [2], [9], [10], [11], [12], [13]). In 1957, Posner [11] initiated the study of identities involving derivations that ensure commutativity. More precisely, the classical Posner's second theorem states that a prime ring must be commutative if it admits a non-zero derivation $d$ satisfying $d(x) x-x d(x) \in Z(R)$ for all $x \in R$. In this direction, Ashraf and Rehman [3] examined the commutativity of a prime ring $R$ admitting a non-zero derivation $d$ that satisfies any one of the conditions: $d(x y) \pm x y \in Z(R), d(x y) \pm y x \in Z(R)$ and $d(x) d(y) \pm x y \in Z(R)$ for all $x, y \in I$, a non-zero ideal of $R$. These results have been proved for generalized derivations in [4]. In [1], Albaş proved the following theorem: Let $R$ be a prime ring with char $(R) \neq 2$. If $R$ admits a generalized derivation $F$ with associated derivation $d$ satisfying $F([x, y])=[F(x), F(y)]$ or $F([x, y])=[F(y), F(x)]$ for all $x, y \in R$, then either $R$ is commutative or $d=0$ or $d=1_{i d}$ or $d=-1_{i d}$, where $1_{i d}$ is the identity map of $R$. Very recently, Huang [10] explored the commutativity of prime rings with specific additive mapping $F$ that satisfy the following identities: (i) $F(x y) \pm x y \in$ $Z(R)$, (ii) $F([x, y]) \pm[F(x), y] \in Z(R)$, (iii) $F([x, y]) \pm[F(x), F(y)] \in Z(R)$, (iv) $[F(x), y] \pm[x, F(y)] \in Z(R)$. Moreover, it would also be interesting and general to investigate the commutativity of prime and semiprime rings with various types of multiplicative derivations. Gusic [9] proved that if $F$ is a multiplicative (generalized)derivation of a prime ring $R$ associated with a non-zero mapping $d$ and $I$ is a nonzero ideal of $R$ such that $F(x y)=F(y) F(x)$ for all $x, y \in I$, then $R$ is commutative. In 2013, Dhara and Ali [7] collected information about the commutative structure of prime and semiprime rings admitting multiplicative (generalized)-derivations that satisfy any one of the conditions: (i) $F(x y) \pm x y \in Z(R)$, (ii) $F(x y) \pm y x \in Z(R)$, (iii) $F(x) F(y) \pm x y \in Z(R)$, (iv) $F(x) F(y) \pm y x \in Z(R)$. In the same line of investigation, Ali et al. [2] obtained many structural results of prime and semiprime rings. Recently, Dhara and Pradhan [8] studied the following left annihilator conditions: (i) $a(F(x y) \pm$ $x y)=0$, (ii) $a(F(x y) \pm y x)=0$, (iii) $a(d(x) F(y) \pm x y)=0$, (iv) $a(d(x) F(y) \pm y x)=0$, (v) $a(F(x) F(y) \pm x y)=0$, (vi) $a(F(x) F(y) \pm y x)=0$ for all $x, y \in I$, where $F$ is a generalized (multiplicative)-derivation, $d$ is the associated multiplicative derivation and $I$ is a non-zero ideal of a prime $\operatorname{ring} R$.

It is known that every noncentral Jordan ideal and every noncentral square-closed Lie ideal of a 2-torsion free semiprime ring $R$ contains a non-zero ideal of $R$ (see [13], [14] resp.). Therefore, in this view it is the optimal case to study certain algebraic 
identities over one-sided ideals of rings. In the present paper, motivated by Huang [10] and Dhara and Pradhan [8], we study a number of central valued conditions with multiplicative (generalized)-derivations on one-sided ideals of prime rings and obtain the commutativity of $R$.

\section{Preliminary Results}

The following basic commutator identities are useful in the sequel.

$$
[x y, z]=x[y, z]+[x, z] y,[x, y z]=y[x, z]+[x, y] z .
$$

We begin our discussion with the following basic lemmas that will be frequently used in our results.

Lemma 2.1 ([10], Lemma 2). Let $R$ be a prime ring. Then for some $0 \neq a \in Z(R)$, if $a b \in Z(R)$, then $b \in Z(R)$. In particular, if $a b=0$, then $b=0$.

Lemma $2.2([5])$. Every prime ring $R$ having a non-zero one sided commutative ideal is commutative.

Lemma 2.3. Let $R$ be a prime ring and $I$ be a non-zero left (resp. right) ideal of $R$. If for any $a, b \in R, a I b=(0)$, then either $a=0$ or $I b=(0)($ resp. $a I=(0)$ or $b=0)$.

Proof. Since $R$ is a prime ring, this fact can be easily obtained.

Lemma 2.4. Let $R$ be a ring and $\delta$ be a multiplicative derivation of $R$. Then $\delta(Z(R)) \subseteq Z(R)$.

Proof. It is trivial to observe that $\delta(0)=0$. Now let $c \in Z(R)$ be any element.

$$
\delta(x c)=\delta(x) c+x \delta(c) \text { and } \delta(c x)=\delta(c) x+c \delta(x) .
$$

Combining these both expressions, we get $[\delta(c), x]=0$ for all $x \in R$. Hence $\delta(c) \in$ $Z(R)$ for all $c \in Z(R)$.

Lemma 2.5. Let $R$ be a prime ring and $\varrho$ be a non-zero right ideal of $R$. If there exists $a \in R$ such that $a \varrho \neq(0)$ and $a[x, y] \in Z(R)$ for all $x, y \in \varrho$, then $R$ is commutative.

Proof. Let us assume that $a[x, y] \in Z(R)$ for all $x, y \in \varrho$. It means $[a[x, y], r]=0$ for all $x, y \in \varrho$ and $r \in R$. It implies that

$$
a[[x, y], r]+[a, r][x, y]=0, \forall x, y \in \varrho, r \in R .
$$

Take $y x$ instead of $y$ in above relation to obtain $a[x, y][x, r]=0$ for all $x, y \in \varrho$ and $r \in R$. It implies that $a[x, y] R[x, r]=(0)$ for all $x, y \in \varrho$ and $r \in R$. Thus for each $x \in \varrho$, either $a[x, \varrho]=(0)$ or $x \in Z(R)$. Therefore in each case we have

$$
a[x, y]=0, \forall x, y \in \varrho .
$$

Take $x s$ for $x$ in last expression, where $s \in R$, we get $a x[s, y]=0$. Since $a \varrho \neq(0)$, by Lemma 2.3, it is straightforward to find that $\varrho \subseteq Z(R)$. Hence $R$ is commutative, by Lemma 2.2. 
Lemma 2.6. Let $R$ be a prime ring and $\varsigma$ be a non-zero left ideal of $R$. If $\sigma: R \rightarrow$ $R$ is a ring automorphism such that $\sigma([x, y]) \in Z(R)$ for all $x, y \in \varsigma$, then $R$ is commutative.

Proof. Let us assume that $\sigma([x, y]) \in Z(R)$ for all $x, y \in \varsigma$. It implies that

$$
[\sigma([x, y]), r]=0, \forall x, y \in \varsigma, r \in R .
$$

Take $x y$ for $y$ in this equation, we find

$$
[\sigma(x), r][\sigma(x), \sigma(y)]=0, \forall x, y \in \varsigma, r \in R .
$$

Replace $r$ by $s r$ such that $s \in R$ in the above expression, we obtain

$$
[\sigma(x), s] R[\sigma(x), \sigma(y)]=(0), \forall x, y \in \varsigma, s \in R .
$$

It implies that for each $x \in \varsigma$, either $[\sigma(x), \sigma(\varsigma)]=(0)$ or $\sigma(x) \in Z(R)$. Hence $[\sigma(x), \sigma(y)]=0$ for all $x, y \in \varsigma$. In view of Lemma $2.2, R$ is commutative.

\section{Main Results}

Theorem 3.1. Let $R$ be a prime ring and $\varrho$ be a non-zero right ideal of $R$. Suppose that $R$ admits multiplicative (generalized)-derivations $(F, f)$ and $(G, g)$ such that one of $f$ and $g$ is non-vanishing on $Z(R)$. If there exists $a \in R$ such that a $\neq \neq(0)$, then the following assertions are equivalent:

(i) $a([F(x), y] \pm[x, G(y)]) \in Z(R)$ for all $x, y \in \varrho$.

(ii) $a(F([x, y]) \pm[G(x), y]) \in Z(R)$ for all $x, y \in \varrho$.

(iii) $a(F([x, y]) \pm[x, G(y)]) \in Z(R)$ for all $x, y \in \varrho$.

(iv) $R$ is commutative.

Proof. $(i) \Rightarrow(i v)$ : Let us first assume that

$$
a([F(x), y]+[x, G(y)]) \in Z(R), \forall x, y \in \varrho .
$$

In case $(0) \neq f(Z(R))$, we choose $c \in Z(R)$ such that $0 \neq f(c)$ and replace $x$ by $x c$ in $(3.1)$ to get

$$
a([F(x), y]+[x, G(y)]) c+a[x f(c), y] \in Z(R), \forall x, y \in \varrho .
$$

Eq. (3.1) gives $a[x f(c), y] \in Z(R)$ for all $x, y \in \varrho$. Since $f$ is a multiplicative derivation (see [6, Lemma 2]), in view of Lemma 2.4, it follows that $a[x, y] f(c) \in Z(R)$ for all $x, y \in \varrho$. Applying Lemma 2.1, it implies that $a[x, y] \in Z(R)$ for all $x, y \in \varrho$. By Lemma $2.5, R$ is commutative. 
Let us now assume that $0 \neq g(Z(R))$. Then we choose $c^{\prime} \in Z(R)$ such that $0 \neq g\left(c^{\prime}\right)$. Substitute $y c^{\prime}$ in place of $y$ in (3.1) to find

$$
a([F(x), y]+[x, G(y)]) c^{\prime}+a\left[x, y g\left(c^{\prime}\right)\right] \in Z(R), \forall x, y \in \varrho .
$$

It implies $a[x, y] g\left(c^{\prime}\right) \in Z(R)$ for all $x, y \in \varrho$. With similar computations as in the case $(0) \neq f(Z(R))$, we get the conclusion.

Notice that if $(G, g)$ is a multiplicative (generalized)-derivation, then so is $(-G,-g)$. Thus by taking $(-G,-g)$ in place of $(G, g)$ in the above proof, we get the same outcome with the identity $a([F(x), y]-[x, G(y)]) \in Z(R)$ for all $x, y \in \varrho$.

$($ ii $) \Rightarrow($ iv $)$ : Let us assume that

$$
a(F([x, y])+[G(x), y] \in Z(R), \forall x, y \in \varrho .
$$

Choose $c \in Z(R)$ such that $0 \neq f(c)$, take $y c$ for $y$ in (3.2) we get

$$
a(F([x, y])+[G(x), y]) c+a[x, y] f(c) \in Z(R), \forall x, y \in \varrho .
$$

It implies that $a[x, y] f(c) \in Z(R)$ for all $x, y \in \varrho$. Since $f$ is a multiplicative derivation, in view of Lemma 2.4 and Lemma 2.1, we find that $a[x, y] \in Z(R)$ for all $x, y \in \varrho$. By Lemma 2.5, we get the conclusion.

We now assume that $0 \neq g(Z(R))$. Then we choose $c^{\prime} \in Z(R)$ such that $0 \neq g\left(c^{\prime}\right)$. Substitute $y c^{\prime}$ in place of $y$ in $(3.2)$, we obtain

$$
a(F([x, y])+[G(x), y]) c^{\prime}+a[x, y] f\left(c^{\prime}\right) \in Z(R), \forall x, y \in \varrho .
$$

Using the hypothesis, we get

$$
a[x, y] f\left(c^{\prime}\right) \in Z(R), \forall x, y \in \varrho .
$$

Substitute $x c^{\prime}$ in place of $x$ in (3.2), we find that

$$
a(F([x, y])+[G(x), y]) c^{\prime}+a[x, y] f\left(c^{\prime}\right)+a\left[x g\left(c^{\prime}\right), y\right] \in Z(R), \forall x, y \in \varrho .
$$

Using the hypothesis, we get

$$
a[x, y] f\left(c^{\prime}\right)+a\left[x g\left(c^{\prime}\right), y\right] \in Z(R), \forall x, y \in \varrho .
$$

Eq. (3.4) and (3.3) gives $a\left[x g\left(c^{\prime}\right), y\right] \in Z(R)$ for all $x, y \in \varrho$. Since $g$ is a multiplicative derivation, we find $a[x, y] g\left(c^{\prime}\right) \in Z(R)$ for all $x, y \in \varrho$. Thus, it follows that $a[x, y] \in$ $Z(R)$ for all $x, y \in \varrho$. By Lemma 2.5, we get the conclusion. In the same way, we get the desired result with $a(F([x, y])-[G(x), y]) \in Z(R)$ for all $x, y \in \varrho$.

$(i i i) \Rightarrow(i v)$ : Let us consider the situation

$$
a(F([x, y])+[x, G(y)]) \in Z(R), \forall x, y \in \varrho .
$$

In case $(0) \neq f(Z(R))$, we choose $c \in Z(R)$ such that $0 \neq f(c)$ and replace $x$ by $x c$ in $(3.5)$ to get

$$
a(F([x, y])+[x, G(y)]) c+a[x, y] f(c) \in Z(R), \forall x, y \in \varrho .
$$


Eq. (3.5) gives $[x, y] f(c) \in Z(R)$ for all $x, y \in \varrho$ and $c \in Z(R)$. Since $f$ is a multiplicative derivation, we find $a[x, y] \in Z(R)$ for all $x, y \in \varrho$. Thus $R$ is commutative, by Lemma 2.5 .

Let us now assume that $(0) \neq g(Z(R))$. Then we choose $c^{\prime} \in Z(R)$ such that $0 \neq g\left(c^{\prime}\right)$. Substitute $y c^{\prime}$ in place of $y$ in $(3.5)$, we find

$$
a(F([x, y])+[x, G(y)]) c^{\prime}+a[x, y] f\left(c^{\prime}\right)+a\left[x, y g\left(c^{\prime}\right)\right] \in Z(R), \forall x, y \in \varrho .
$$

Using the hypothesis, we get

$$
a[x, y] f\left(c^{\prime}\right)+a\left[x, y g\left(c^{\prime}\right)\right] \in Z(R), \forall x, y \in \varrho .
$$

Substitute $x c^{\prime}$ in place of $x$ in (3.5), we find that

$$
a(F([x, y])+[x, G(y)]) c^{\prime}+a[x, y] f\left(c^{\prime}\right) \in Z(R), \forall x, y \in \varrho .
$$

Using the hypothesis, we get

$$
a[x, y] f\left(c^{\prime}\right) \in Z(R), \forall x, y \in \varrho .
$$

Eq. (3.6) and (3.7) gives $a[x, y] g\left(c^{\prime}\right) \in Z(R)$ for all $x, y \in \varrho$. Since $g$ is a multiplicative derivation, $a[x, y] \in Z(R)$ for all $x, y \in \varrho$. In view of Lemma 2.5, $R$ is commutative. In the same way, we get the desired conclusion from $a(F([x, y])-[x, G(y)] \in Z(R)$ for all $x, y \in \varrho$.

Corollary 3.2. Let $R$ be a prime ring and $I$ be a non-zero ideal of $R$. Suppose that $R$ admits multiplicative (generalized)-derivations $(F, f)$ and $(G, g)$ such that one of $f$ and $g$ is non-vanishing on $Z(R)$. For some $0 \neq a \in R$, the following assertions are equivalent:

(i) $a([F(x), y] \pm[x, G(y)]) \in Z(R)$ for all $x, y \in I$.

(ii) $a(F([x, y]) \pm[G(x), y]) \in Z(R)$ for all $x, y \in I$.

(iii) $a(F([x, y]) \pm[x, G(y)]) \in Z(R)$ for all $x, y \in I$.

(iv) $R$ is commutative.

Proof. The proof is straightforward.

Theorem 3.3. Let $R$ be a prime ring and $\varsigma$ be a non-zero left ideal of $R$. Suppose that $R$ admits multiplicative (generalized)-derivations $(F, f),(G, g)$ and a ring automorphism $\sigma$ such that one of $f$ and $g$ is non-vanishing on $Z(R)$. Then the following assertions are equivalent:

(i) $\sigma([F(x), y] \pm[x, G(y)]) \in Z(R)$ for all $x, y \in \varsigma$.

(ii) $\sigma(F([x, y]) \pm[G(x), y]) \in Z(R)$ for all $x, y \in \varsigma$.

(iii) $\sigma(F([x, y]) \pm[x, G(y)]) \in Z(R)$ for all $x, y \in \varsigma$. 
(iv) $R$ is commutative.

Proof. $(i) \Rightarrow(i v)$ : Let us consider the situation

$$
\sigma([F(x), y]+[x, G(y)]) \in Z(R), \forall x, y \in \varsigma .
$$

In case $(0) \neq f(Z(R))$, we choose $c \in Z(R)$ such that $0 \neq f(c)$ and replace $x$ by $c x$ in (3.8) to get

$$
\sigma([F(x), y]+[x, G(y)]) \sigma(c)+\sigma([x f(c), y]) \in Z(R), \forall x, y \in \varsigma .
$$

Eq. (3.9) gives that $\sigma([x f(c), y]) \in Z(R)$ for all $x, y \in \varsigma$. In view of Lemma 2.4, we have $\sigma([x, y]) \sigma(f(c)) \in Z(R)$ for all $x, y \in \varsigma$. Since $0 \neq f(c)$ and $\sigma$ is injective, so $\sigma(f(c)) \neq 0$. It follows from Lemma 2.1 that

$$
\sigma([x, y]) \in Z(R), \forall x, y \in \varsigma .
$$

By Lemma 2.6, $R$ is commutative.

We now assume that $(0) \neq g(Z(R))$. Let us choose $c^{\prime} \in Z(R)$ such that $0 \neq g\left(c^{\prime}\right)$. Replace $y$ with $c^{\prime} y$ in (3.8), we get

$$
\sigma([F(x), y]+[x, G(y)]) \sigma\left(c^{\prime}\right)+\sigma\left(\left[x, y g\left(c^{\prime}\right)\right]\right) \in Z(R), \forall x, y \in \varsigma .
$$

Using the hypothesis, we obtain $\sigma\left(\left[x, y g\left(c^{\prime}\right)\right]\right) \in Z(R)$ for all $x, y \in \varsigma$. In view of Lemma 2.4, we have

$$
\sigma([x, y]) \sigma\left(g\left(c^{\prime}\right)\right) \in Z(R), \forall x, y \in \varsigma .
$$

Since $0 \neq \sigma\left(g\left(c^{\prime}\right)\right)$, we get $\sigma([x, y]) \in Z(R)$ for all $x, y \in \varsigma$. In view of Lemma 2.6, we get our conclusion. In the same way, we get commutativity of $R$ from the condition $\sigma([F(x), y]-[x, G(y)]) \in Z(R)$ for all $x, y \in \varsigma$.

$(i i) \Rightarrow(i v)$ : We now assume that

$$
\sigma(F([x, y])+[G(x), y]) \in Z(R), \forall x, y \in \varsigma .
$$

Choose $c \in Z(R)$ such that $0 \neq f(c)$, take $y c$ for $y$ in (3.11) in order to obtain

$$
\sigma(F([x, y])+[G(x), y]) \sigma(c)+\sigma([x, y]) \sigma(f(c)) \in Z(R), \forall x, y \in \varsigma .
$$

Eq. (3.11) gives $\sigma([x, y]) \sigma(f(c)) \in Z(R)$ for all $x, y \in \varsigma$. By same reasoning as above, we have $\sigma([x, y]) \in Z(R)$ for all $x, y \in \varsigma$. By Lemma 2.6, $R$ is commutative.

Assume that $(0) \neq g(Z(R))$. By our assumption there exists $c^{\prime} \in Z(R)$ such that $0 \neq g\left(c^{\prime}\right)$. Replace $y$ by $y c^{\prime}$ in $(3.11)$, we get $\sigma(F([x, y])+[G(x), y]) \sigma\left(c^{\prime}\right)+$ $\sigma([x, y]) \sigma\left(f\left(c^{\prime}\right)\right) \in Z(R)$ for all $x, y \in \varsigma$. By the given hypothesis, we have $\sigma([x, y]) \sigma\left(f\left(c^{\prime}\right)\right) \in Z(R)$ for all $x, y \in \varsigma$. Take $x c^{\prime}$ instead of $x$ in (3.11) and using it, we may infer that $\sigma([x, y]) \sigma\left(f\left(c^{\prime}\right)\right)+\sigma\left(\left[x g\left(c^{\prime}\right), y\right]\right) \in Z(R)$ for all $x, y \in \varsigma$. Further it reduces to $\sigma\left(\left[x g\left(c^{\prime}\right), y\right]\right) \in Z(R)$ for all $x, y \in \varsigma$. Applying Lemma 2.4, we get $\sigma([x, y]) \sigma\left(g\left(c^{\prime}\right)\right) \in Z(R)$ for all $x, y \in \varsigma$. In view of Lemma 2.1, we obtain 
$\sigma([x, y]) \in Z(R)$ for all $x, y \in \varsigma$. Hence $R$ is commutative, by Lemma 2.6. Similarly, we can get the results when $\sigma(F([x, y]-[G(x), y])) \in Z(R)$ for all $x, y \in \varsigma$.

$($ iii $) \Rightarrow(i v)$ : Let us consider

$$
\sigma(F([x, y])+[x, G(y)]) \in Z(R), \forall x, y \in \varsigma .
$$

In case $(0) \neq g(Z(R))$, we choose $c \in Z(R)$ such that $0 \neq g(c)$ and replace $y$ by $y c$ in (3.12) we have

$$
\sigma(F([x, y])+[x, G(y)]) \sigma(c)+\sigma([x, y]) \sigma(f(c))+\sigma([x, y g(c)]) \in Z(R), \forall x, y \in \varsigma .
$$

Substitute $x c$ in place of $x$ in (3.12), we find that

$$
\sigma(F([x, y])+[x, G(y)]) \sigma(c)+\sigma([x, y]) \sigma(f(c)) \in Z(R), \forall x, y \in \varsigma .
$$

Eq. (3.13) and (3.14) gives $\sigma([x, y]) \sigma(g(c)) \in Z(R)$ for all $x, y \in \varrho$. It follows from above that $\sigma([x, y]) \in Z(R)$ for all $x, y \in \varrho$. With the aid of Lemma 2.6, we are done.

We next assume that $(0) \neq f(Z(R))$. One may notice that the similar implications as in the above case ensure the conclusion, therefore we omit the details. In the same way, we get the conclusion from $\sigma(F([x, y])-[x, G(y)]) \in Z(R)$ for all $x, y \in \varsigma$.

With the similar arguments as in the proof of Theorem 3.1 with necessary modifications, we obtain the following result and for the sake of brevity, we omit its proof.

Theorem 3.4. Let $R$ be a prime ring and $\varrho$ be a non-zero right ideal of $R$. Suppose that $R$ admits multiplicative (generalized)-derivations $(F, f)$ and $(G, g)$ such that $f$ and $g$ are both non-vanishing on $Z(R)$. If there exists some $a \in R$ such that $a \varrho \neq(0)$, then the following assertions are equivalent:

(i) $a(F([x, y]) \pm[G(x), F(y)]) \in Z(R)$ for all $x, y \in \varrho$.

(ii) $a(F([x, y]) \pm[F(x), G(y)]) \in Z(R)$ for all $x, y \in \varrho$.

(iii) $R$ is commutative.

Corollary 3.5. Let $R$ be a prime ring and $I$ be a non-zero ideal of $R$. Suppose that $R$ admits multiplicative (generalized)-derivations $(F, f)$ and $(G, g)$ such that $f$ and $g$ are both non-vanishing on $Z(R)$. Then for some $0 \neq a \in R$, the following assertions are equivalent:

(i) $a(F([x, y]) \pm[G(x), F(y)]) \in Z(R)$ for all $x, y \in I$.

(ii) $a(F([x, y]) \pm[F(x), G(y)]) \in Z(R)$ for all $x, y \in I$.

(iii) $R$ is commutative

Theorem 3.6. Let $R$ be a prime ring and $\varsigma$ be a non-zero left ideal of $R$. Suppose that $R$ admits a ring automorphism $\sigma$ and multiplicative (generalized)-derivations $(F, f)$ and $(G, g)$ such that $f$ and $g$ are both non-vanishing on $Z(R)$. Then the following assertions are equivalent: 
(i) $\sigma(F([x, y]) \pm[G(x), F(y)]) \in Z(R)$ for all $x, y \in \varsigma$.

(ii) $\sigma(F([x, y]) \pm[F(x), G(y)]) \in Z(R)$ for all $x, y \in \varsigma$.

(iii) $R$ is commutative.

Proof. $(i) \Rightarrow($ iii $)$ : Let us first consider the situation $\sigma(F([x, y])+[G(x), F(y)]) \in$ $Z(R)$ for all $x, y \in \varsigma$. By applying the same technique that we used in the proof of Theorem 3.4, we get $\sigma([x, y]) \in Z(R)$ for all $x, y \in \varsigma$. By Lemma 2.6, we are done. Similarly, we conclude in case $\sigma(F([x, y])-[G(x), F(y)]) \in Z(R)$ for all $x, y \in \varsigma$.

$($ ii $) \Rightarrow($ iii $)$ : Proof is omitted.

Theorem 3.7. Let $R$ be a prime ring and $\varsigma$ be a non-zero left ideal of $R$. Suppose that $R$ admits multiplicative (generalized)-derivations $(F, f)$ and $(G, g)$ such that both $f$ and $g$ are non-vanishing on $Z(R)$. Then for some $0 \neq a \in R$, the following assertions are equivalent:

(i) $a(F(x) G(y) \pm x y) \in Z(R)$ for all $x, y \in \varsigma$.

(ii) $a(F(x) G(y) \pm y x) \in Z(R)$ for all $x, y \in \varsigma$.

(iii) $R$ is commutative.

Proof. $(i) \Rightarrow($ iii $)$ : We first consider the case

$$
a(F(x) G(y)+x y) \in Z(R), \forall x, y \in \varsigma .
$$

In view of our assumption, let us choose $c \in Z(R)$ such that $0 \neq g(c)$. Take $y c$ for $y$ in $(3.15)$ to get

$$
a(F(x) G(y)+x y) c+a F(x) y g(c) \in Z(R), \forall x, y \in \varsigma .
$$

By the given hypothesis, it implies that $a F(x) y g(c) \in Z(R)$ for all $x, y \in \varsigma$. Since $0 \neq g(c)$, by Lemma 2.1, we have $a F(x) y \in Z(R)$ for all $x, y \in \varsigma$. That is

$$
[a F(x) y, r]=0, \forall x, y \in \varsigma, r \in R .
$$

Take $x t$ in place of $x$ in $(3.16)$, we obtain

$$
[a F(x) t y, r]+[a x f(t) y, r]=0, \forall x, y, t \in \varsigma, r \in R .
$$

Eq. (3.16) reduces it to

$$
[a x f(t) y, r]=0, \forall x, y, t \in \varsigma, r \in R .
$$

Replace $x$ by $a x$ in the above expression and using it to get

$$
[a, r] a x f(t) y=0, \forall x, y, t \in \varsigma, r \in R .
$$

It implies that $[a, r] \operatorname{Rax} f(t) y=(0)$ for all $x, y, t \in \varsigma$ and $r \in R$. Thus we either have $a \in Z(R)$ or $a x f(t) y=0$ for all $x, y, t \in \varsigma$. Suppose that $a x f(t) y=0$ for 
all $x, y, t \in \varsigma$. Since $0 \neq a$, it implies that $x f(t) y=0$ and hence $x f(t)=0$ for all $x, t \in \varsigma$. Choose $c_{1} \in Z(R)$ such that $f\left(c_{1}\right) \neq 0$ and substitute $t c_{1}$ instead of $t$, we have $c_{1} x f(t)+x f\left(c_{1}\right) t=0$ for all $x, t \in \varsigma$. Then it is not difficult to see that $x f\left(c_{1}\right)=0$ for all $x \in \varsigma$. By Lemma 2.4, we have $f\left(c_{1}\right) x=0$. Take $r x$ for $x$, where $r \in R$, we get that $f\left(c_{1}\right) R x=(0)$ for all $x \in \varsigma$. It implies that either $f\left(c_{1}\right)=0$ or $x=0$, but none of them is true, and hence a contradiction follows.

Thus we have $a \in Z(R)$. By Lemma 2.1, from (3.15) we find that $F(x) G(y)+x y \in$ $Z(R)$ for all $x, y \in \varsigma$. By repeating the similar arguments as above, we arrive at the situation $[x f(t) y, r]=0$ for all $x, y, t \in \varsigma$ and $r \in R$. Replace $x$ with $q x$, where $q \in R$, we obtain that $[q, r] x f(t) y=0$ for all $x, y, t \in \varsigma$ and $r, q \in R$. It implies that $[q, r] \operatorname{Rxf}(t) y=(0)$, and hence either $[q, r]=0$ for all $r, q \in R$ or $x f(t) y=0$ for all $x, y, t \in \varsigma$. Clearly the latter case is not possible, thus we have $[R, R]=(0)$. Similarly, we can conclude that $R$ is commutative in case $a(F(x) G(y)-x y) \in Z(R)$ for all $x, y \in \varsigma$.

$($ ii $) \Rightarrow($ iii $)$ : With the similar implications, we can easily prove this part, therefore we omit its proof.

We conclude this discussion with the following example, which shows that the hypotheses of our results are not superfluous.

Example 3.8. Let $S$ be a ring. Consider

$$
R=\left\{\left[\begin{array}{cccc}
0 & a & b & c \\
0 & 0 & 0 & d \\
0 & 0 & 0 & e \\
0 & 0 & 0 & 0
\end{array}\right] \mid a, b, c, d, e \in S\right\}
$$

Define maps $F, f, G, g: R \rightarrow R$ by

$$
\begin{aligned}
& F\left(\left[\begin{array}{llll}
0 & a & b & c \\
0 & 0 & 0 & d \\
0 & 0 & 0 & e \\
0 & 0 & 0 & 0
\end{array}\right]\right)=\left[\begin{array}{cccc}
0 & 0 & 0 & b^{2} \\
0 & 0 & 0 & 0 \\
0 & 0 & 0 & e \\
0 & 0 & 0 & 0
\end{array}\right], \\
& f\left(\left[\begin{array}{cccc}
0 & a & b & c \\
0 & 0 & 0 & d \\
0 & 0 & 0 & e \\
0 & 0 & 0 & 0
\end{array}\right]\right)=\left[\begin{array}{cccc}
0 & 0 & 0 & b d \\
0 & 0 & 0 & 0 \\
0 & 0 & 0 & 0 \\
0 & 0 & 0 & 0
\end{array}\right], \\
& G\left(\left[\begin{array}{cccc}
0 & a & b & c \\
0 & 0 & 0 & d \\
0 & 0 & 0 & e \\
0 & 0 & 0 & 0
\end{array}\right]\right)=\left[\begin{array}{cccc}
0 & 0 & 0 & 0 \\
0 & 0 & 0 & b d^{2} \\
0 & 0 & 0 & 0 \\
0 & 0 & 0 & 0
\end{array}\right], \\
& g\left(\left[\begin{array}{cccc}
0 & a & b & c \\
0 & 0 & 0 & d \\
0 & 0 & 0 & e \\
0 & 0 & 0 & 0
\end{array}\right]\right)=\left[\begin{array}{cccc}
0 & 0 & 0 & a b c \\
0 & 0 & 0 & 0 \\
0 & 0 & 0 & 0 \\
0 & 0 & 0 & 0
\end{array}\right] .
\end{aligned}
$$


It can be easily verified that $(F, f)$ and $(G, g)$ are multiplicative (generalized)derivations of $R$. Note that

$$
Z(R)=\left\{\left[\begin{array}{llll}
0 & 0 & 0 & c \\
0 & 0 & 0 & 0 \\
0 & 0 & 0 & 0 \\
0 & 0 & 0 & 0
\end{array}\right] \mid c \in S\right\}
$$

and the set

$$
\varrho=\left\{\left[\begin{array}{cccc}
0 & 0 & b & c \\
0 & 0 & 0 & -b \\
0 & 0 & 0 & 0 \\
0 & 0 & 0 & 0
\end{array}\right] \mid b, c \in S\right\}
$$

is a right ideal of $R$. One can check that none of our hypotheses are true, i.e., $R$ is not a prime ring, $f$ and $g$ are both vanishing on $Z(R)$ and there exists $x \in \varrho$ such that $a x=0$ for all $a \in R$. We note that the following identities are satisfied:

(i) $a([F(x), y] \pm[x, G(y)]) \in Z(R)$.

(ii) $a([F(x), y] \pm[G(x), y]) \in Z(R)$.

(iii) $a(F([x, y]) \pm[x, G(y)]) \in Z(R)$.

(iv) $a(F([x, y]) \pm[G(x), F(y)]) \in Z(R)$.

(v) $a(F([x, y]) \pm[F(x), G(y)]) \in Z(R)$.

(vi) $a(F(x) G(y) \pm x y) \in Z(R)$.

(vii) $a(F(x) G(y) \pm y x) \in Z(R)$.

for all $x, y \in \varrho$. However, $R$ is not commutative.

\section{Open Problems}

The following are some natural questions, that we are unable to answer at that moment:

1. How to remove the condition that the associated derivations are non-vanishing on $Z(R)$ ?

2. Is there any example which shows that this assumption can not be relaxed to get the given results? 


\section{References}

[1] E. Albas, On generalized derivations satisfying certain identities, Ukrain. Math. J. 63 (1) (2011) 596 - 602. DOI: 10.1007 /s11253-011-0535-7.

[2] A. Ali, B. Dhara, S. Khan, F. Ali, Multiplicative (generalized)-derivations and left ideals in semiprime rings, Hacettepe J. Math. Stat. 44 (6) (2015) 1293-1306.

[3] M. Ashraf, N. Rehman, On derivations and commutativity in prime rings, EastWest J. Math. 3 (1) (2001) $87-91$.

[4] M. Ashraf, A. Ali, S. Ali, Some commutativity theorems for rings with generalized derivations, Southeast Asian Bull. Math. 31 (2007) 415 - 421.

[5] H.E. Bell, W.S. Martindale III, Centralizing mappings of semiprime rings, Canad. Math. Bull. 30 (1) (1987) 92 - 101. DOI: 10.4153 /CMB-1987-014-x.

[6] D.K. Camci, N. Aydin, On multiplicative (generalized)-derivations in semiprime rings, Commun. Fac. Sci. Univ. Ank. Ser. A1 Math. Stat. 66 (1) (2017) 153-164. DOI:10.1501/Commua1_0000000784.

[7] B. Dhara, S. Ali, On multiplicative (generalized)-derivations in prime and semiprime rings, Aequat. Math. 86 (2013) 65 - 79. DOI: 10.1007/s00010-013$0205-y$.

[8] B. Dhara, K.G. Pradhan, A note on multiplicative (generalized)-derivations with annihilator conditions, Georgian Math. J. 23 (2) (2016) 191 - 198. DOI:10.1515/gmj-2016 - 0020 .

[9] I. Gusić, A note on generalized derivations of prime rings, Glasnik Mate. 40 (60) (2005) $47-49$.

[10] S. Huang, Generalized reverse derivations and commutativity of prime rings, Commun. Math. 27 (2019) 43 - 50. DOI:10.2478/cm-2019-0004.

[11] E.C. Posner, Derivations in prime rings, Proc. Amer. Math. Soc. 8 (1957) $1093-$ 1100. DOI: $10.2307 / 2032686$.

[12] N. Rehman, R.M. Al-Omary, N.M. Muthana, A note on multiplicative (generalized) $(\alpha, \beta)$-derivations in prime rings, Annales Math. Silesianae 33 (2019) $266-275$.

[13] G.S. Sandhu, D. Kumar, A note on derivations and Jordan ideals of prime rings, AIMS Math. 2 (4) (2017) 580 - 585. DOI:10.3934/Math.2017.4.580; correction 4 (3) (2019) 684-685. DOI:10.3934/Math.2019.3.684.

[14] G.S. Sandhu, D. Kumar, Derivations satisfying certain algebraic identities on Lie ideals, Math. Morav. 23 (2) (2019) 79 - 86. DOI:10.5937/MatMor1902079S. 
DOI: $10.7862 /$ rf.2021.7

\section{Gurninder S. Sandhu}

email: gurninder_rs@pbi.ac.in

ORCID: 0000-0001-8618-6325

Department of Mathematics

Patel Memorial National College

Rajpura-140401, Punjab

INDIA

\section{Ayşe Ayran}

email: ayseayran@stu.comu.edu.tr

ORCID: 0000-0002-1626-5498

Department of Mathematics

Çanakkale Onsekiz Mart University

Çanakkale-17020

TURKEY

\section{Neşet Aydin}

email: neseta@comu.edu.tr

ORCID: 0000-0002-7193-3399

Department of Mathematics

Çanakkale Onsekiz Mart University

Çanakkale-17020

TURKEY

Received 26.11.2020

Accepted 13.05.2021 